\title{
MAGNETIC FIELDS IN WHITE DWARFS
}

\author{
Gary D. Schmidt \\ Steward Observatory, University of Arizona \\ Tucson, Arizona 85721, USA
}

1. Introduction

Just one year ago at the 2nd Conference on Faint Blue Stars, two review papers were presented summarizing the observational characteristics and attempts at spectral modeling of isolated magnetic white dwarfs (see Schmidt 1987 and Wickramasinghe 1987, respectively). Because much of that information is still relevant, this paper will concentrate on some of the progress which has occurred since the time of that meeting, and on highlighting current problems in the area of research. Using specific examples for illustration, the discussion concentrates on the single stars. Magnetic binary systems are considered from the point of view of the white dwarfs themselves.

\section{Recent Identifications and the Current Magnetic Sample}

During the past year, two new white dwarfs were added to the list of known magnetic stars: a DA with a polar field of $\sim 24 \mathrm{MG}\left(1 \mathrm{MG}=10^{6} \mathrm{G}\right)$, found in the course of a QSO survey (Foltz and Latter 1988), and a unique DC + magnetic DQ wide binary (Ruiz and Maza 1988, also reported at this meeting). As summarized in Table 1 , this brings the current sample to a total of 26 stars spanning the range in field strength $\sim 1-1000$ MG.

Magnetic candidates are drawn from a variety of sources. Prior to the advent of electronic array detectors, spectroscopy was generally not adept at distinguishing diffuse, shallow features, so many of the early magnetic stars were identified through the continuum circular polarization which is induced by the field (e.g. Kemp 1970; Angel 1977). The polarization arises because of circular dichroism, which at low and intermediate fields, resembles a splitting of the continuum opacity functions for opposite handed modes of propagation of the electric vector. As shown in Fig. 1, this effect results in a fractional optical polarization of $V \approx 1 \%$ at a field of 50 MG (dependent also on opacity source and field orientation). Above $100 \mathrm{MG}$, however, the simple Zeeman analogy breaks down as the opacity sources depart substantially from their zero-field functional form and propagation modes become elliptical. Linear polarization then begins to appear, again dependent on geometry, but amounting to $P \approx 1 \frac{\circ}{\circ}$ at a strength of $200 \mathrm{MG}$ and 
climbing rapidly thereafter (Fig 1.). Clearly, the sensitivity of polarimetric surveys is limited to strong magnetic fields.

Fortunately, magnetic sensitivities of the low members of the hydrogen Balmer series fall in the range 5-15A/MG, so surface strengths as weak as $10 \mathrm{MG}$ are easily recognized in most modern survey-quality spectroscopy. This is the origin of many of the recent discoveries, esp. the PG sample. Of course, for DC spectral types or in field strength regimes where features are severely smeared, spectroscopy loses its effectiveness (see, however, the discrete features of $H$ in PG1031+234 [Latter, Schmidt, and Green 1987], at nearly $10^{9} \mathrm{MG}$ ).

Comparatively weak fields, 105-6 $\mathrm{G}$, should be detectable in principle through wavelength shifts of the high-order Balmer lines resulting from the quadratic Zeeman effect (e.g., Preston 1970). Values another factor of 10 smaller might be recognized as subtle broadening of the non-LTE cores of $H \alpha$ in the cooler DAs, assuming the profile could be distinguished from rotational smearing. The idea of recognizing comparatively weak magnetic fields through "magnetoseismology" has also been recently suggested by Jones et al. (1988, and summarized by Kawaler at this meeting), but considering the paucity of pulsating white dwarfs, this technique is not likely to be of assistance in discovering significant numbers of low-field stars.

Characteristic Polarization of MWDs

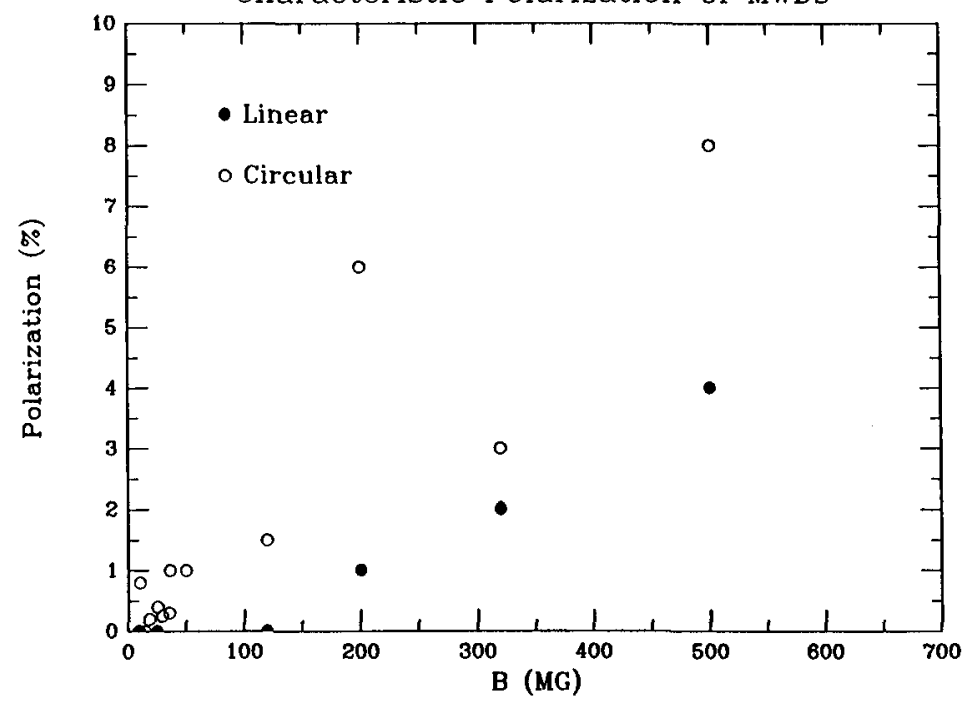

Fig. 1. The observed characteristic levels of continuum circular and linear polarization in the optical for the stars listed in Table 1. Although temperature, opacity source, viewing angle and field structure cause a wide dispersion in the degree of polarization at any given field value, the general trends are useful for estimating field strengths on stars with featureless or unidentified spectra. 
TABLE 1

ISOLATED MAGNETIC WHITE DWARFS

\begin{tabular}{|c|c|c|c|c|c|c|}
\hline Object & $\begin{array}{c}T \\
(K)\end{array}$ & $\begin{array}{l}\text { Spectral } \\
\text { features }\end{array}$ & Period & $\begin{array}{l}\langle P\rangle \\
\left(\frac{8}{8}\right)\end{array}$ & $\begin{array}{l}\langle V\rangle \\
\left(\frac{o}{b}\right)\end{array}$ & $\begin{array}{r}\text { Bp } \\
(M G)\end{array}$ \\
\hline $\begin{array}{l}\text { PG } 0136+251 \\
\text { PG } 1658+441 \\
\text { G141-2 }\end{array}$ & $\begin{array}{r}45000 \\
30000 \\
5600\end{array}$ & $\begin{array}{l}\mathrm{H} \\
\mathrm{H} \\
\mathrm{H}\end{array}$ & & & & $\begin{array}{r}2 \\
3.5 \\
5\end{array}$ \\
\hline $\begin{array}{l}\text { GD90 } \\
\text { G } 99-37\end{array}$ & $\begin{array}{r}12000 \\
6200\end{array}$ & $\mathrm{C} 2, \stackrel{\mathrm{H}}{\mathrm{CH}}$ & & $<.1$ & $\begin{array}{r}<.15 \\
.8\end{array}$ & $\begin{array}{r}9 \\
10\end{array}$ \\
\hline KUV 03292+0035 & 15000 & H & & & & 12 \\
\hline $\begin{array}{ll}\text { GD } & 356 \\
\text { HS } & 1254+3430\end{array}$ & $\begin{array}{r}7500 \\
15000\end{array}$ & $H\left(e_{H}\right)$ & & & $<.05$ & $\begin{array}{l}15 \\
15\end{array}$ \\
\hline KPD $0253+5052$ & 20000 & $\mathrm{H}$ & $4.1 \mathrm{~h}$ & & .2 & 18 \\
\hline $1136-014$ & & $\mathrm{H}$ & & & & 24 \\
\hline G $99-47$ & 5700 & $\mathrm{H}$ & & $<.1$ & .4 & 25 \\
\hline KUV 813-14 & 10400 & H & & & .25 & 29 \\
\hline$P G \quad 1533-057$ & 20000 & H & & & & 31 \\
\hline Feige 7 & 22000 & $\mathrm{H}, \mathrm{He}$ & $2.2 \mathrm{~h}$ & & .3 & 35 \\
\hline BPM 25114 & 20000 & H & $2.8 d$ & & 1 & 36 \\
\hline PG $1313+095$ & 15000 & H & $5.4 \mathrm{~h}$ & & 1 & $\sim 50$ \\
\hline GD 116 & 15000 & $\mathrm{H}$ & & & & 65 \\
\hline ESO $439-162$ & 6300 & C2 & & & & $\sim 100$ \\
\hline G 195-19 & 8000 & none & $1.3 d$ & $<.13$ & .8 & $\sim 100$ \\
\hline PG $1015+015$ & 10000 & H & $1.6 \mathrm{~h}$ & & 1 & 120 \\
\hline G $227-35$ & 7000 & $?$ & & .25 & 2 & $\sim 150$ \\
\hline LP $790-29$ & 8600 & $\mathrm{C} 2$ & & 1 & 6 & 200 \\
\hline C $240-72$ & 6000 & $?$ & & 1 & .5 & $\sim 200$ \\
\hline GD 229 & 16000 & $?$ & & 4 & $<.2$ & $>200$ \\
\hline GrW +70 8247 & 14000 & $H$ & & 2 & 3 & 320 \\
\hline PG $1031+234$ & 15000 & $\mathrm{H}$ & $3.4 \mathrm{~h}$ & 4 & 8 & 500 \\
\hline
\end{tabular}

Taking together the early searches for magnetic fields (see the historical perspective in Liebert 1988), line profile studies, surveys for rotation (e.g. Pilachowski and Milkey 1987), and the now-popular quest for short-period double-degenerate binaries, a reasonable estimate might be that the line spectra of $\sim 1 / 3$ of known white dwarfs have been observed with sufficient dispersion and signal-to-noise ratio that fields in the range $\sim 105-6 \mathrm{G}$ would have been discovered. Yet, none have. The current definite detection limit is represented by PC $1658+441$ at a polar value of $\sim 3.5 \mathrm{MG}$, and $P G 0136+251$ is a probable magnetic star with a strength about a factor of two weaker (Liebert et al. 1983). Thus, it would appear that the distribution of degenerates with surface field strength as presented by Schmidt (1987) is a reasonable approximation to reality: overall, the magnetic sample (1-1000 MG) comprises no more than $\sim 2-3 \%$ of the total number of known white dwarfs, and the broad distribution shows a moderate but probably significant preference for the range $\sim 10-100 \mathrm{MG}$. We conclude that by far the majority of white dwarfs possess fields below a surface strength of $10^{5} \mathrm{G}$.

The distribution of white dwarf primary stars with magnetic field strength is an important topic of discussion among Cataclysmic Variable (CV) enthusiasts, since 
a strong field can dictate such basic features as the dynamics of accretion, the emitted energy distribution, spin/orbit synchronization, and probably even the long-term evolution of the system. For the purposes of this conference, the situation can be summarized as follows: field strengths on about half of the AM Her systems have been directly measured to fall in the range 20-55 MG, and the remainder of these 15 synchronized binaries are probably very similar. Another dozen or so systems, called the DQ Her binaries or Intermediate Polars, show coherent optical and $X$-ray brightness modulations which are associated with a magnetic field on the rotating primary star, but the field is too weak (or the system too large) to disrupt the accretion disk and synchronize the spin and orbital motions. The spectrum of near-infrared circular polarization detected from one such binary confirms its magnetic nature and suggests a surface field value of $\sim 5-10$ MC (West et al. 1987).

Not only are very strong-field $(B>100 \quad M G)$ primaries conspicuous in their absence among known CVs, but weak and intermediate-field systems are far more numerous than their counterparts in the field. More than $20 \%$ of all known CVs are magnetic, and the AM Her and DQ Her binaries mentioned above comprise more than $1 / 3$ of all systems with orbital periods shorter than two hours. Although some of the discrepancy vis-a-vis field degenerates can be attributed to the enhanced $X$-ray luminosity (therefore enhanced detectability) resulting from magnetically channeled accretion, it is unlikely that this is an order-of-magnitude effect. One avenue for consideration is the effect that a strong magnetic field might have on the production of a short-period binary during the common-envelope phase of evolution.

\section{Field Morphology}

Wickramasinghe (1987) provides an excellent recent review of the relevant physics and attempts at spectral modeling of fields on the known magnetic white dwarfs. In general, the distribution of field strength over the photosphere is best deduced from the profiles of Zeeman-displaced absorption lines, while the run of circular and linear polarization through a line and/or the rotational modulation of continuum polarization can be used to infer field direction. Since hydrogen is the only element for which reasonably complete and accurate Zeeman calculations exist (e.g., Henry and O'Connell 1985; Wunner et al. 1987), such work has thus far been confined to the magnetic DAs.

The recent study of Grw $+70^{\circ} 8247$ by Wickramasinghe and Ferrario (1988) is a particularly credible exercise if only because of the wealth of high quality data on this bright, well-observed star. Modeling of both the rich line spectrum as well as 
circular and linear spectropolarimetry were attempted, with the result that a pole-on dipole of $320 \mathrm{MG}$ polar strength yielded reasonably good fits to the data. However, particular problems were noted in matching features longward of $\sim 6000 \AA$ and the polarimetric profiles of the lines. S. Jordan, in a paper presented at this meeting, applies a similar approach to $\mathrm{Grw}+70^{\circ}$, and obtains somewhat better agreement with the red transitions but even poorer polarimetric profiles. No doubt a portion of this difficulty results from the fact that, although the Zeeman pattern of hydrogen is reasonably well in hand, the atmospheric physics and opacity functions in fields as strong as those on Grw $+70^{\circ} 8247$ are not currently tractable (Wickramasinghe 1987). Until those problems are resolved, it is difficult to contemplate substantial improvements to the models for very high-field objects.

Perhaps most informative are the results for the rotating magnetic stars (see Fig. 2). As an example, Wickramasinghe and Cropper (1988) have recently studied phase-resolved spectroscopy and spectropolarimetry of the 100 MG star PC 1015+015. which rotates with a period of $99 \mathrm{~m}$. At this field strength the use of shifted zero-field opacity curves is more secure, and the opportunity to study a full range of viewing angles greatly reduces ambiguities in the possible geometries. Unfortunately, the star is sufficiently faint that the polarimetry was of little use aside from defining the continuum behavior with rotational phase. The authors conclude that the field morphology approximates that of a centered dipole with polar strength of $\sim 120 \mathrm{MG}$ which is oriented roughly orthogonal to the spin axis of the star.

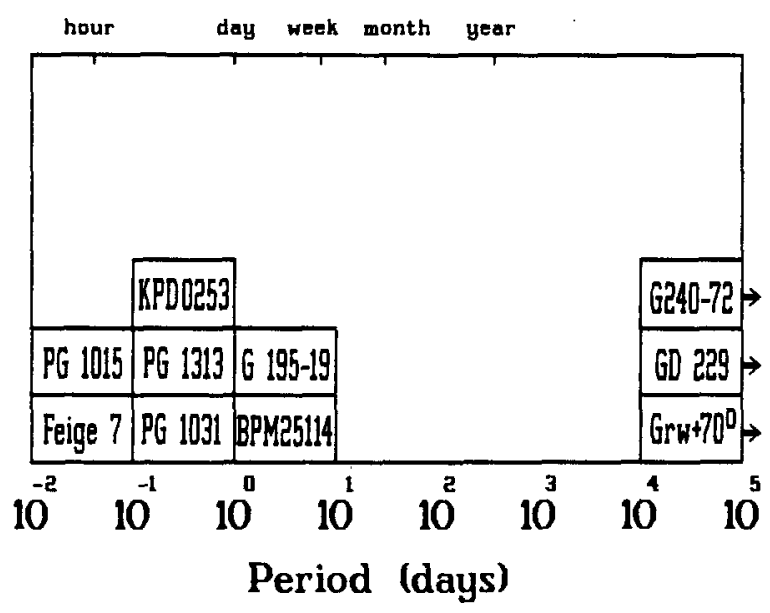

Fig. 2. Distribution of rotation periods deduced from periodic variations in the circular polarization of magnetic white dwarfs. The very long periods shown for a few stars are based on the assumption of an oblique rotator and the absence of detectable variations in polarization over more than a decade of monitoring. 
It should not be inferred from the above examples that the field patterns on all white dwarfs are dipolar in nature. The most strongly magnetized white dwarf identified to date, PG $1031+234$, exhibits a hydrogen Zeeman pattern which depicts fields between 200 and nearly $1000 \mathrm{MG}$ through its $3^{\mathrm{h}} 24^{\mathrm{m}}$ rotation period (Schmidt et al. 1986). Although the overall structure on the surface of this star suggests a dipole with a polar strength in the vicinity of 500 MG (Latter et al. 1987), the most intense fields are found in a single magnetic "spot". In attempting to model PG 1031+234, Schmidt et al. (1986) found the distortions to the phase-resolved continuum polarization curve caused by this spot so severe that the ignorance of high-field atomic physics was deemed a second-order effect.

Although the rather simple field morphologies deduced for the majority of magnetic stars studied to date would seem to indicate the presence of well-organized underlying global structures, white dwarfs are sufficiently dense that even hundred-MG fields can be anchored in a relatively thin skin. Such an explanation has been proffered for the magnetic "spot" on PG 1031+234, and chromospheric activity has been proposed to account for the apparently rather tangled field on surface of the unique magnetic emissionline degenerate GD 356 (Greenstein and McCarthy 1985).

\section{The Magnetic Nova V1500 Cygni}

The recent discovery that Nova Cygni 1975 occurred in a magnetic binary system (now designated $\mathrm{V} 1500 \mathrm{Cyg}$ ) provides a unique opportunity to study the effects which a strong magnetic field has on the nova event (and, perhaps ultimately vice versa). Among known novae, the 1975 event was an exceedingly fast eruption: rising at least 19 magnitudes above the POSS plate limit to an apparent visual magnitude of $V=1.8$ and declining by 3 mags in just 4 days. At maximum light, the absolute visual magnitude of $M V=-10.2$ marked Nova Cygni as one of the brightest of such events. These facts, in part, have led to the conclusion that the mass of the white dwarf primary in this binary is nearly at the Chandrasekhar limit (see the recent review by Lance, McCall, and Uomoto 1988).

The post-nova decline was marked by peculiar brightness oscillations on a timescale somewhat longer than 3 hours. Well-observed by Patterson (1979) and others, the period of these variations decreased by more than 4 minutes in the year following outburst; when observations resumed following the winter of 1976, the period had stabilized at an intermediate value of $3^{\mathrm{h}} 21^{\mathrm{m}}$. The fluctuations have continued at that period with a fixed ephemeris to the current mean brightness of $V$ $=17.1$ and amplitude $\sim 0.5-1 \mathrm{mag}$. Although the oscillations were generally thought to reflect rotational or orbital motion, the aperiodicity was not readily explained at the time. 
In 1987, V1500 Cyg was found to exhibit optical circular polarization modulated on a period some 3.5 minutes shorter than the post-1977 photometric period (Stockman, Schmidt, and Lamb 1988). Like the AM Her systems, this was interpreted by the authors to indicate the emission of optical cyclotron radiation in a magnetic field $>10 \mathrm{MG}$ from the vicinity of the accreting post-nova primary. The polarimetric period then reflects the rotation period of the magnetic white dwarf; the only reasonable explanation for the post-1977 stable photometric oscillation is the cyclical appearance of the inner heated hemisphere of the tidally-locked corotating secondary star.

A binary period of $3^{\mathrm{h}} 21^{\mathrm{m}}$ places $\mathrm{V} 1500$ Cyg near the long-period extreme of the phase-locked systems, but among other AM Her binaries. Moreover, the presence of a magnetic field sufficient to give rise to optical cyclotron emission and the near-synchronism of $\mathrm{V} 1500 \mathrm{Cyg}$ even after the nova outburst suggested to Stockman et al. that the binary was indeed a synchronized AM Her system prior to eruption. The proposed process by which it became uncoupled involves frictional angular momentum transfer (e.g. MacDonald 1980) from the orbiting secondary to the primary while the white dwarf was bloated beyond the size of binary system (see Stockman et al.). The authors show that the concept of a magnetic pole-accretor provides a ready explanation for the entire post-eruption photometric history of the system (Fig. 3).

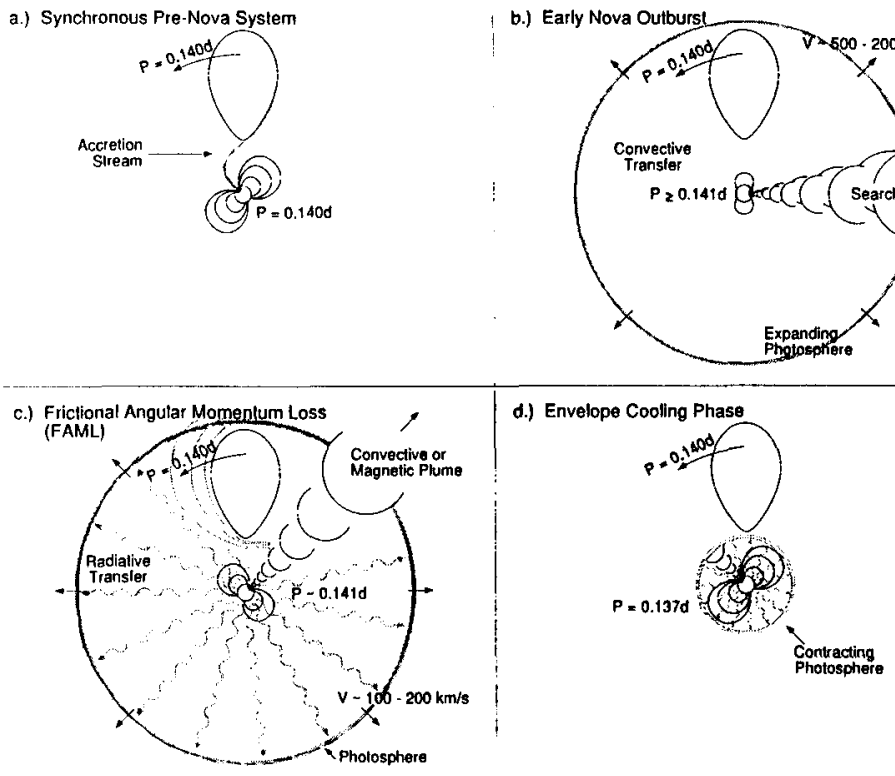

Fig. 3. An explanation of the remarkable photometric variations observed during the first few years following the eruption of Nova Cygni 1975. The system begins the process as a phase-locked magnetic AM Her system, accreting primarily onto one magnetic pole. Decoupling of the spin and orbital periods occurs due to frictional angular momentum transfer between the secondary and primary star when the atmosphere is distended beyond the extent of the binary system. 
The mechanism by which spin/orbit synchronization occurs in a magnetic $C V$ is a topic of popular concern, since the phenomenon is also linked to the orbital period evolution of the binary. For fields typical of AM Her variables, estimates of the timescale for phase-locking generally fall in the range $10^{3}-10^{5}$ years; indeed, $\vee 1500$ Cyg must resynchronize during that period if it is to avoid a period runaway due to future nova events. For these timescales, the required time derivative of the rotational period falls in the range

$$
7 \times 10^{-9}>\dot{P}>7 \times 10^{-11} \text {. }
$$

respectively. The past year of polarimetric study of V1500 Cyg by the author and collaborators has yielded a rotational ephemeris accurate to better than 0.1 sec, and the timing residuals from strict periodicity provide a limit of $\dot{P}<4 \times 10^{-9}$ (Fig. 4). Thus, the data already exclude phase-locking on a timescale of $10^{3}$ years. With the sensitivity scaling as (time) $^{2}$, there is good reason to believe that continued monitoring over the next few years will directly test synchronizing theories.

The magnetic field strength on $\mathrm{V} 1500 \mathrm{Cyg}$ has not yet been measured. Due to its distance and intervening absorption, the system is rather faint, and with the current light output dominated by the heated secondary, searches for Zeeman features due to the white dwarf's photosphere are unpractical. An attempt is currently underway to obtain a crude estimate of the field strength through measurement of the spectrum of polarized cyclotron flux. With this in hand, many aspects of the eruption and postnova behavior of $\mathrm{V} 1500 \mathrm{Cyg}$ will be placed on a more quantitative footing.

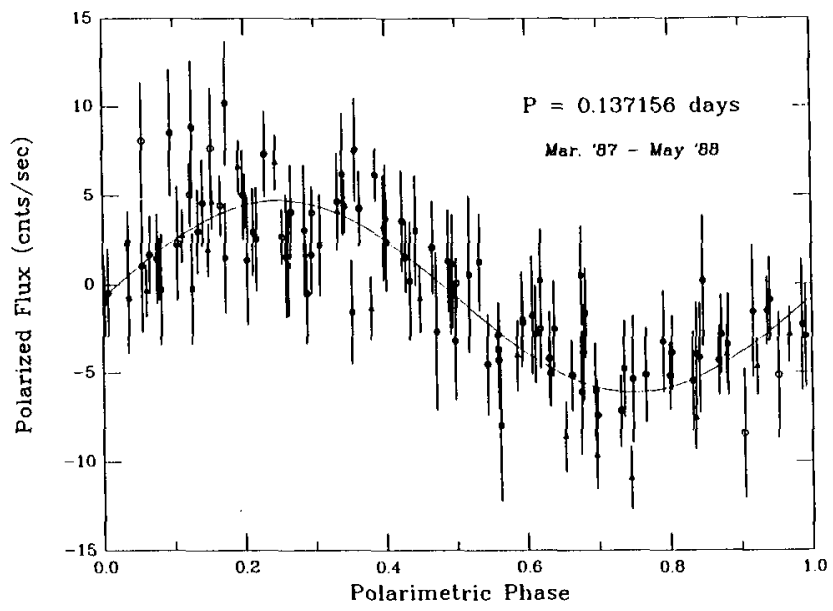

Fig. 4. One year of polarimetric monitoring of $\vee 1500$ Cyg phased on a single period. Different symbols represent various epochs of observation. The uncertainty in period is less than 0.1 sec; timing residuals from strict periodicity imply $\dot{P}<4 \times 10^{-9}$. 
The author is especially grateful to C. Foltz, M. Ruiz, and J. Maza for ensuring the timeliness of this review by providing information on recent magnetic discoveries prior to their publication. The recent developments on the unique magnetic nova system V1500 Cyg presented here are to be included in a future publication (Schmidt and Stockman 1988). Research by the author on magnetic white dwarfs and magnetic binary systems is supported, in part, by the National Science Foundation through grant AST 86-19296.

\section{References}

Angel, J. R. P. 1977, Ap. J., 216, 1.

Foltz, C. B., and Latter W. B. 1988, private communication.

Greenstein, J. L. and McCarthy, J. K 1985, Ap. J., 289, 732.

Henry, R. J. W., and O'Connell, R. F. 1985, Pub. A. S. P., 97, 333.

Jones, P. W., Pesnell, W. D., Kawaler, S. D., and Hansen, C. J. 1988, preprint.

Kemp, J. C. 1970, Ap. J., 162, 169.

Lance, C. M., McCall, M. L., and Uomoto, A. K. 1988, Ap. J. Suppl., in press.

Latter, W. B., Schmidt, G. D., and Green, R. F. 1987, Ap. J., 320, 308.

Liebert, J., Schmidt, G. D., Green, R. F., Stockman, H. S., and McGraw, J. T. 1983, Ap. J., 264, 262.

Liebert, J. 1988, Pub. A. S. P., in press.

MacDonald, J. 1980, M. N. R. A. S., 191, 933.

Patterson, J. 1979, Ap. J., 231, 789.

Pilachowski, C., and Milkey, R. W. 1987, Pub. A. S. P., 99, 836.

Preston, C. W. 1970, Ap. J. Lett., 160, L143.

Ruiz, M. T. and Maza, J. 1988, preprint.

Schmidt, G. D. 1987, in The Second Conference on Faint Blue Stars, proc. IAU Colloquium No. 95, ed. A.G.D. Philip, D. S. Hayes, and J. W. Liebert (Schenectady: L. Davis Press), pp. 377-388.

Schmidt, G. D., and Stockman, H. S. 1988, in preparation.

Schmidt, G. D., West, S. C., Liebert, J., Green, R. F., and Stockman, H. S. 1986, Ap. J., 309, 218.

Stockman, H. S., Schmidt, G. D., and Lamb, D. Q. 1988, Ap. J., in press.

West, S. C., Berriman, G., and Schmidt, G. D. 1987, Ap. J. (Letters), 322, L35.

Wickramasinghe, D. T. 1987, in The Second Conference on Faint Blue Stars, proc. IAU

Colloquium No. 95, ed. A.G.D. Philip, D. S. Hayes, and J. W. Liebert (Schenectady: L. Davis Press), pp. 389-400.

Wickramasinghe, D. T., and Cropper, M. 1988, preprint.

Wickramasinghe, D. T., and Ferrario, L. 1988, Ap. J., 327, 22.

Wunner, G., Geyer, F., and Ruder, H. 1987, Astrophys. and Sp. Sci., 131, 595. 